\title{
Tumor markers panel and tumor size of ovarian dermoid tumors in reproductive age
}

\author{
Var T, Tonguc EA, Ugur M, Altinbas S, Tokmak A \\ Zekai Tahir Burak Women's Health Research and Education Hospital, Department of Reproductive Endocrinology, \\ Ankara, Turkey. drturgutvar@yahoo.com
}

\begin{abstract}
Objective: To study whether there is any relationship between a serum tumor markers panel (CA19-9, CA 125, CEA, CA15-3 and AFP) and the tumor size in patients with ovarian dermoid cyst in reproductive age. Background: Between January 2006-2007, 160 patients were operated in the Department of Infertility, Zekai Tahir Burak Women Health Hospital due to ovarian dermoid cyst. Methods: The clinical data and serum tumor markers levels of operated patients were retrieved from the records of the department. This was a retrospective study.

Results: Forty-four patients (37.6\%) had high levels of CA 19-9, twenty-seven patients (19.3 \%) had high CA 125 levels, eleven patients $(9.4 \%)$ had high levels of CEA, five patients $(4 \%)$ had high levels of CA $15-3$ and one patient $(0.9 \%)$ had high AFP levels at the time of initial surgery. The bilaterality rate was $8.1 \%$. When grouping the tumor size as $<4 \mathrm{~cm}, 4.1-10 \mathrm{~cm}$ and $>10 \mathrm{~cm}$, the mean serum levels of tumor markers had significantly increased by increasing the tumor size $(p<0.05)$ for CA 19-9, CA 125, CEA.

Conclusions: Our study suggests that serum CA $19-9$ is probably more accurate marker than other tumor markers in the ovarian dermoid cysts. Also, the most important parameter that affects CA 19-9 elevation in the dermoid cysts, is the tumor size. As the tumor becomes bigger, this relationship becomes more distinct (Tab. 2 , Ref. 15). Full Text in PDF www.elis.sk.

Key words: ovarian dermoid tumors, tumor markers, tumor size, reproductive age, CA 19-9.
\end{abstract}

Tumor markers are widely used in the differential diagnosis of adnexial masses. CA125 elevations are noted mostly in the epithelial ovarian neoplasm and endometrioma. Whereas the mucinous tumors are commonly associated with the elevation of CA19-9, CA15-3, which is widely used in the breast cancer, they are not commonly used in the diagnosis of ovarian pathologies. Other tumor markers such as AFP are elevated in case of the ovarian germ cell tumors (1).

The ovarian dermoid cysts are among the most commonly encountered germ cell tumors of the ovary. Although they are almost always benign, malignant transformation has been reported to occur in $2 \%$. Eighty-eight percent of the cases are unilateral, and $80 \%$ of them are detected during the reproductive period. While dermoid cysts make up to $27-44 \%$ of all primary ovarian tumors, they form $35-58 \%$ of the benign tumors (2). Benign conditions of the ovary may also be associated with the elevation of the aforementioned tumor markers.

The aim of this study was to determine the role of the tumor markers CA 19-9, CA 125, CEA, CA 15-3 and AFP in patients with dermoid cysts, as well as to study their correlation with tumor size.

Zekai Tahir Burak Women's Health Research and Education Hospital, Department of Reproductive Endocrinology, Ankara, Turkey

Address for correspondence: T. Var, MD, Tunali Hilmi cad.64/4 Kavaklidere/Ankara 06100, Turkey.

Phone: +905323719862, Fax: +903124266453

\section{Materials and methods}

The clinical notes of 207 patients who underwent surgery for the ovarian dermoid cysts at the Departments of Infertility in our hospital between January 2006 and January 2007 were evaluated retrospectively. Forty seven patients were excluded from the study if any of the following situations occurred: 1) Pregnancy, 2) Concomitant diseases (especially hepatic or renal), 3) additive pathology in operation (e.g. myomas, endometriosis), 4) high biochemical parameters, and 5) malignant transformation. Institutional ethics board's approval was obtained for this study. Written informed consent was obtained from all patients. This study was conducted in accordance with basic principles of Helsinki Declaration.

All patients underwent preoperative transabdominal and transvaginal ultrasonographic examination. Blood samples were obtained from the patients with ultrasound findings consistent with the ovarian cysts for the determination of preoperative serum levels CA19-9, CA 125, CA 15-3, CEA and AFP. The blood samples were taken from the patients who have neither eaten nor smoked for 12 hours in the early follicular phase of the menstruation.

Taking the ultrasonographic tumor size into consideration, the patients underwent laparoscopic/ laparatomic cystectomy. Histopathologic examination took place at the Department of Pathology.

Serum levels of CA 125, CA 19-9, AFP, CA 15-3 and CEA were analyzed using an automated micro particle enzyme immunoassay (Roche Diagnostics, Germany). The upper normal limits (UNL) for the cancer antigens CA 125, CA 19-9 and CA 15-3 
were $35 \mathrm{U} / \mathrm{ml}, 37 \mathrm{U} / \mathrm{ml}$, and $30 \mathrm{U} / \mathrm{ml}$, respectively. For CEA, the upper normal limit was $4 \mathrm{ng} / \mathrm{ml}$, while for AFP it was $11 \mathrm{ng} / \mathrm{ml}$.

Patients with elevated CA 15-3 were evaluated with mammography and breast ultrasound, as well as being consulted with a general surgeon, to rule out any breast pathology. Similarly, patients with CA 19-9 elevations were referred to a gastroenterologist for gastrointestinal system evaluation. There were no breast or gastrointestinal pathology.

The tumor size was analyzed as reported during the operation. For patients with bilateral lesions, the sum of the dimensions of both cysts was taken into consideration. Other parameters that were included in the final analysis were the patient age, parity, presenting complaint, menstrual history, smoking status and tumor localization.

\section{Statistical Analysis}

Data analysis was performed by using the SPSS for Windows, version 11.5 (SPSS Inc., Chicago, IL, United States). Whether the distributions of continuous variables were normal or not was determined by using the Shapiro Wilcoxon test. Levene test was used for the evaluation of homogeneity of variances. Continuous data were shown as the mean \pm standard deviation or median (IQR), where applicable. Categorical data were presented as number of cases and percentages.

The differences between two independent groups regarding for tumor markers were compared by the Mann-Whitney U test. When the number of independent groups was more than two, the Kruskal-Wallis test was used. When the p-value from the Kruskal-Wallis tests was statistically significant, to know which group differ from which other, the multiple comparison test was used.

Categorical data were analyzed by the Chi-square or Fisher's exact test, where appropriate.

Whether the statistically significant effects of tumor size on higher CA19-9 according to the univariate analyses were going on or not was evaluated by the Multiple Logistic Regression Analysis. The odds ratio and $95 \%$ CIs for each independent variables were also calculated.

The $\mathrm{p}$ value less than 0.05 was considered as statistically significant.

\section{Results}

The patients included in the study had the mean age of $29.01 \pm$ 6.38 (range: 17-42). Among the tumor markers, the elevations in CA 19-9 levels were the most commonly encountered in 44

Tab. 1. The median tumor markers values with regard to tumor size.

\begin{tabular}{lcccc}
\hline \multicolumn{5}{c}{ Tumor size $(\mathrm{cm})$} \\
\hline Variables & $<4 \mathrm{~cm}$ & $4-10 \mathrm{~cm}$ & $>10 \mathrm{~cm}$ & $\mathrm{p}$ \\
\hline $\mathrm{CA} 19-9>37(\mathrm{U} / \mathrm{ml})$ & $20.16 \pm 17.9$ & $84.0 \pm 15.8$ & $295 \pm 180$ & $0.001^{\mathrm{a}}$ \\
$\mathrm{CA} 125>35(\mathrm{U} / \mathrm{ml})$ & $21.5 \pm 14.5$ & $24.6 \pm 18.8$ & $60.5 \pm 52.6$ & $0.001^{\mathrm{a}}$ \\
$\mathrm{CEA}>4(\mathrm{ng} / \mathrm{ml})$ & $1.3 \pm 0.6$ & $2.0 \pm 1.2$ & $7.2 \pm 2.7$ & $0.001^{\mathrm{a}}$ \\
$\mathrm{CA} 15-3>30(\mathrm{U} / \mathrm{ml})$ & $17.5 \pm 9.1$ & $16.0 \pm 8.5$ & $16.8 \pm 7.3$ & $\mathrm{NS}$ \\
$\mathrm{AFP}>11(\mathrm{U} / \mathrm{ml})$ & $1.7 \pm 0.7$ & $1.9 \pm 1.8$ & $1.5 \pm 0.6$ & $\mathrm{NS}$ \\
\hline${ }^{\mathrm{a}} \mathrm{p}<0.05$ is considered to be statistically significant. Data are expressed as the \\
mean $\pm \mathrm{SD}$.
\end{tabular}

Tab. 2. The number and rate of patients with elevated tumor markers with regard to tumor size.

\begin{tabular}{lcccc}
\hline \multicolumn{5}{l}{ The number and rate of patients with elevated tumor markers } \\
\hline Variables & $<4 \mathrm{~cm}(\mathrm{n}=33)$ & $4-10 \mathrm{~cm}(\mathrm{n}=114)$ & $>10 \mathrm{~cm}(\mathrm{n}=13)$ & $\mathrm{p}$ \\
\hline CA19-9 & $3(11.5)$ & $35(39.3)$ & $6(60)$ & $0.008 \mathrm{a}$ \\
CA125 & $2(7.1)$ & $17(17)$ & $8(66.7)$ & $0.001 \mathrm{a}$ \\
CEA & $0(0)$ & $7(8.4)$ & $4(44.4)$ & $0.001 \mathrm{a}$ \\
CA15-3 & $2(8)$ & $3(3.4)$ & $0(0)$ & NS \\
AFP & $0(0)$ & $1(1.3)$ & $0(0)$ & NS \\
\hline
\end{tabular}

${ }^{a} \mathrm{p}<0.05$ is considered to be statistically significant. Data are expressed as $\mathrm{n}(\%)$.

(37.6 \%) patients. This was followed by the elevations in CA-125, CEA and CA 15-3 in 27 (19.3\%), 9 (11\%) and 5 (3.1\%) patients, respectively. Only 1 patient had elevated AFP. Thirteen patients (9.6\%) had elevations in both CA 19-9 and CA 125 levels, while only 1 patient had elevated levels of all three cancer antigens. Elevations in more than three of the tumor markers were not observed. Fifty-eight patients ( $45 \%$ ) had elevated levels of at least one of CA 19-9 and CA 125 , and 60 patients (48\%) had elevated levels of CA 19-9, CA 125 or CEA.

The mean CA 19-9 level was $87.7 \pm 180.8 \mathrm{U} / \mathrm{ml}(0.1-1000 \mathrm{U} /$ $\mathrm{ml}$ ) and 20 patients had highly elevated CA 19-9, 12 patients had greater than $100 \mathrm{U} / \mathrm{ml}$ and 8 patients had greater than $500 \mathrm{U} / \mathrm{ml}$ ( 2 of them were $1000 \mathrm{U} / \mathrm{ml}$ ).

The mean tumor size was $6.65 \pm 3.14 \mathrm{~cm}$ (range: $2-22$ ). When patients were evaluated in 3 groups according to tumor size, 33 patients had a tumor less than $4 \mathrm{~cm}$ in size, 114 patients had tumors $4-10 \mathrm{~cm}$ in size, and 13 patients had tumors larger than $10 \mathrm{~cm}$.

In the dermoid cysts with a tumor size less than $4 \mathrm{~cm}$, the median values in all tumor markers were below cut-off values. In the tumors between 4-10 cm, a meaningful elevation was observed only in the CA 19-9 level ( $\mathrm{p}=0.001)$. In those larger than $10 \mathrm{~cm}$, a statistically meaningful elevation existed in CA 19-9, CA125 and CEA levels ( $\mathrm{p}=0.001, \mathrm{p}=0.001, \mathrm{p}=0.001$ ) (Tab. 1). When CA 19-9, CA 125 and CEA levels were analyzed based on groups, it was observed that higher levels were associated with bigger tumors $(\mathrm{p}<0.05)$.

CA19-9 elevation was observed in only 3 patients $(11.5 \%)$ with a tumor less than $4 \mathrm{~cm}$ in size, and in 35 patients $(39.3 \%)$ with a tumor size between $4-10 \mathrm{~cm}$. Of the 10 patients with a tumor bigger than $10 \mathrm{~cm}, 6(60 \%)$ had elevated CA 19-9. These results among the three groups were statistically meaningful $(\mathrm{p}=0.008)$ (Tab. 2). Table 2 also shows the number and the rate of patients with other elevated tumor markers regarding the tumor size.

The site of the tumors was similar on both the right and left ( $48.8 \%$ and $43.1 \%$ respectively). Only $8.1 \%$ of patients had bilateral tumors. Interestingly, only elevations in CA 125 and CA 19-9 occurred in these patients, with the other markers safely within normal range. Furthermore, although bilateral cases had higher CA 125 and CA 19-9 levels when compared to those with unilateral tumors, this difference was statistically not significant.

Seventy one point three percent $(114 / 160)$ of patients were nulliparous, while another $20.8 \%$ (46/160) were multiparous. Seventy six point two percent (122/160) reported having normal menstrual periods at the time of diagnosis, while $23.8 \%$ (38/160) complained 
of irregular menstruation. The vast majority of patients (86.3 \%) (138/160) were non-smoker. Although $17.5 \%$ (28/160) of patients were asymptomatic, gynecologic symptoms (e.g. lower abdominal pain, menstrual irregularities) and infertility were encountered in $73.7 \%(118 / 160)$, and $8.8 \%(14 / 160)$ of patients, respectively.

Regarding the CA 19-9, which was the tumor marker most closely associated with dermoid cysts, we performed a logistic regression analysis that may had an effect on CA 19-9 levels. In this analysis, the age, gravidity, smoking status, patient complaint and tumor size were all evaluated, and the only associations established were with tumor size bigger than $10 \mathrm{~cm}$ (OR:11.252; 95\%CI: 1.893-66.879; $\mathrm{p}=0.008$ ).

\section{Discussion}

Our findings showed that the ovarian dermoids are commonly associated with elevation in the tumor markers especially CA 19-9. The other tumor markers such as CA 125, AFP, CA 15-3 and CEA were also noted, however these are not as prominent as CA19-9.

Ovarian germ cell tumors are the most important cause of elevated AFP in adult women, besides pregnancy (1). In a study by Kawai, elevated AFP was not detected in any of 31 cases of the ovarian dermoid cyst (3). In our study, only 1 patient had elevated AFP, perhaps supporting our suggestion that AFP is not a valuable marker for the dermoid cysts.

Elevations of CA 15-3 have been reported in up to $20 \%$ of benign ovarian pathologies (1). However, in our study only $4 \%$ of patients had elevated CA $15-3$, which leads us to think that it may not be very helpful in dermoid cysts.

CEA is a non specific tumor marker and elevation may occur in a variety of conditions. Smoking is also known to cause elevations in otherwise healthy individuals (1). However, in our study we could not establish a link between cigarette smoking and CEA. In a study by Konishi, CEA was elevated in $30 \%$ of the ovarian dermoid cyst cases, while Kawai and Kikkawa failed to establish a meaningful association (3-5). In our study, elevated CEA was detected in $9.4 \%(11 / 160)$ of patients, although this elevation was found to be associated with a bigger tumor size. Our study confirmed that CEA plays a very limited role in ovarian dermoid cyst.

CA 125 remains the most studied marker in association with the ovarian pathologies. In studies by Kawai, Kikkawa and Mikuni, CA 125 has been shown to increase in the ovarian dermoid cyst, with elevations reported in $23.7 \%, 28 \%$ and $12.7 \%$ of patients, respectively $(3,5,6)$. This rate was $19.3 \%$ in our study, a figure consistent with those from literature. We suggest that CA 125 seems to be useful in the ovarian dermoid cyst.

CA 19-9 is a pure carbohydrate antigen, and is secreted by many different tissues. The specificity of CA 19-9 is limited because elevations above the upper cut off are known to occur in several benign conditions with marked increases reported in acute and chronic pancreatitis (7-11). While Mikuni et al reported CA 19-9 elevations in $45.5 \%$ of dermoid cases, Kikkawa et al observed a slightly higher elevation rate of $59 \%(5,6)$. In our study, this figure was $37.6 \%$, and our study confirms that CA 19-9 has an important role in the diagnosis of dermoid cysts.
In the literature, sixty percent of dermoid cysts are between $5-10 \mathrm{~cm}$ in size; however, up to $10 \%$ may exceed $15 \mathrm{~cm}(12,13)$. In Kikkawa and Dede's studies, the mean tumor sizes were 8.8 $\mathrm{cm}$ and $7.2 \mathrm{~cm}$, respectively $(5,14)$. In our study, the mean tumor size was $6.65 \pm 3.14 \mathrm{~cm}$ and $71.3 \%$ of patients had tumors $4-10$ $\mathrm{cm}$ in size, while $8.1 \%$ were bigger than $10 \mathrm{~cm}$.

Studies in literature have only rarely focused on the tumor size and tumor marker elevation in the ovarian dermoid cysts. In a study by Mikuni, tumor weight and CA 19-9 elevation was examined and no statistically meaningful relationship was found (6). Similarly, Dede studied tumor size and CA 19-9 elevation and found no statistically meaningful difference (14). In our study, all tumor markers and tumor size was studied and a significant relationship was detected between the CA19-9 elevation and tumor diameter. While mean CA 19-9 and other tumor marker levels in dermoid cyst with a tumor under $4 \mathrm{~cm}$ were within normal boundaries, the tumors between $4-10 \mathrm{~cm}$ only had a significant elevation in the CA 19-9 level. Whenever the tumor was bigger than $10 \mathrm{~cm}$, the elevation was observed in CA 19-9, CA125 and CEA levels. However, while CA 125 and CEA elevations were approximately twice as much as normal values, CA19-9 elevation was as much as 8 -fold. The logistical regression analysis showed that a tumor size bigger than $10 \mathrm{~cm}$ was the most important parameter that affected CA 19-9 elevation. There is presently no other study in the literature that examined elevated tumor markers with regard to grouped tumor size.

The bilaterality of dermoid cysts has been extensively studied in literature, with rates reported between $8-15 \%(12,15)$. Our study finding of $8.1 \%$ is consistent with the literature. However, although the link between CA 19-9 and CA 125 elevations and bilaterality has been established by Dede et. al., we could not demonstrate a statistically significant correlation (14). As determined by Mikuni and Ayhan, as well as in our study, the site of tumors was similar on both right and left $(6,12)$.

Elevated tumor marker levels (even at the highest levels) may not always be an indicator of malignancy. Seriously elevated CA 19-9 levels are not a rare finding in the dermoid cysts. Also, in our study, patients who were diagnosed with dermoid cysts had values around $1000 \mathrm{U} / \mathrm{ml}$.

Therefore, if a patient within the reproductive period has a unilateral mobile mass on bimanual examination and a mass bigger than $4 \mathrm{~cm}$ with suspicious findings on ultrasonography, elevations in CA 19-9 may be more suggestive of a dermoid cyst than other ovarian pathologies.

\section{Conclusion}

CA 19-9 is the most valuable tumor marker in dermoid cysts. CA125 may be useful but CEA, CA 15-3 and AFP do not have any significance in the dermoid cysts. The most important parameter that affects CA 19-9 elevation in the dermoid cysts was the tumor size. As the tumor become bigger, this relationship become more distinct. CA 19-9 was the only elevated tumor marker when the tumor diameter was approximately $4-10 \mathrm{~cm}$. In dermoid cysts bigger than $10 \mathrm{~cm}$, the CA 19-9 level was significantly elevated. 
$95-98$

No relationship was found between the CA 19-9 elevation and bilaterality. Future studies are needed to examine the relationship between the tumor size and tumor markers in the dermoid cysts.

\section{References}

1. Patsner B. Tumor markers in Gynecologic Oncology. Copeland Lj (Eds). Textbook of Gynecology. Philedelphia: WB Saunders, 1993.

2. Nanayakkara S, Ali S, Gilmour K. Incresed serum carcinomic antigen 19-9 (CA19-9) in a dermoid cyst. J Obstet Gynecol 2007; 27: 96-97.

3. Kawai M, Kano T, Kikkawa F, Marikawa Y, Oguchi H, Nakashima $\mathbf{N}$ et al. Seven tumor markers in benign and malignant germ cells tumors of the ovary. Gynecol Oncol 1992; 45: 248-253.

4. Konishi I, Fujii S, Okamura H, Sakahara H, Endo K, Torizuka K et al. Analysis of serum CA125, CEA, AFP, LDH levels and LDH isoenzymes in patients with ovarian tumors-correlation between tumor markers and histological types of ovarian tumors. Acta Obstet Gynaecol Jpn 1986; 38: $827-836$.

5. Kikkawa F, Nawa A, Tamakoshi K, Ishikawa H, Kuzuya K, Suganuma $\mathbf{N}$ et al. Diagnosis of squamosus cell carsinoma arising from mature cystic teratoma of the ovary. Cancer 1998; 82: 2249-2255.

6. Mikuni M, Makinoda S, Tanaka T. Evaluation of tumor markers in ovarian dermoid cyst. Acta Obstet Gynecol Jpn 1990; 42: 479-484.

7. Fateh-Moghadam A, Stieber P. Tumor marker und ihr sinnvoller. Einsatz. 2nd eds. Marloffstein-Rathsberg: Jurgen Hartmann Verlag,1993: 36-37.
8. Helfrich G, Klapdor U, Bahlo M, Klapdor R, Schreiber HW. Determination of tumor markers CA 19-9, CA 125, CA 15-3, CA 50 and CEA in acute and chronic benign diseases in medical-surgical patient. Klapdor R (Eds). New tumor markers and monoclonal antibodies. Stutgard - New York, Georg Thieme Verlag, 1987; 287-290.

9. Koch OM, Wust G. Unspesific elevation of tumor marker profiles in severe benign disease. Klapdor R (Eds). New tumor markers and monoclonal antibodies. Stuttgart - New York, Georg Thieme Verlag, 1987; 291-296.

10. Nagata H, Takahashi K, Yamane Y, Yoshino K, Shibukawa T, Kitao M:Abnormally high values of CA 125, CA 19-9 in women with benign tumors. Gynecol Obstet Invest 1989; 28: 165-816.

11. Atabekoglu C, Bozaci EA, Tezcan S. Elevated carbohydrate antigen 19-9 in a dermoid cyst. Int J Gynecol Obstet 2005; 91: 262-263.

12. Ayhan A, Bukulmez O, Genc C, Karamursel SB, Ayhan A. Mature cystic teratomas of the ovary: case series from one institution over 34 years. Eur J Obstet Gynecol and Reprod Biol 2000; 88 (2): 153-157.

13. Caruso PA, Marsh MR, Minkowitz S, Karten G. An intense clinicopathologic study of 305 teratomas of the ovary. Cancer 1971; 27: 343-348.

14. Dede M, Güngör S, Yenen MC, Alanbay I, Duru NK, Haşimi A. CA 19-9 may have clinical significance in mature cystic teratomas of the ovary. Int J Gynecol Cancer 2006; 16: 189-193.

15. Pepe F, Panella M, Pepe G, Panella P, Pennisi F, Arikan S. Dermoid cyst of the ovary. Eur J Gynecol Oncol 1986; 7: 186-191.

Received February 25, 2010. Accepted December 18, 2011. 